\title{
Restriktionen falsch gesetzt
}

\author{
Umweltpolitik steht immer noch im Verdacht, das Wirtschaftswachstum zu \\ begrenzen. Insbesondere Umweltzertifikate oder Lizenzen gelten als Instru- \\ mente zur ökologischen Deckelung des Wachstums. Tatsächlich aber erfolgt \\ dies durch die monetaristische Geldmengenpolitik. Die "Wachstumslizenzen" \\ der Zentralbank halten die Volkswirtschaft in einer Situation der Unter- \\ beschäftigung, bei der wirksame Schritfe in Richfung Nachhaltigkeit politisch \\ keine Chance haben. Eine fundamentale Änderung der geldpolitischen Strategie \\ ist eine nołwendige Bedingung für den ökologischen Strukturwandel.
}

\section{$\mathrm{D}$} Von Gerbard Maier-Rigaud ie Ökonomie als Wissenschaft steht jedweden Grenzen des Wachstum skeptisch gegenüber. Immerhin waren es Ökonomen, die spätestens seit dem 18. Jahrhundert und damit lange vor Darwin den Gedanken der Entwicklung geistesgeschichtlich eingeführt haben. Die Evolution wurde durch den Einfluß der Ökonomie allgemein zum Paradigma der Wissenschaften. Und schließlich hat die industrielle Revolution den eindrucksvollen empirischen Beleg für wirtschaftliche Entwicklungsmöglichkeiten geliefert. Im Theoriegebäude der Ökonomie kommen Grenzen nicht vor. Gegenstand dieser Disziplin sind relative Knappheiten und dementsprechend relative Preise. Auf lange Sicht ist alles substituierbar: Arbeit, Kapital und natïrliche Ressourcen. Abnehmende Grenzerträge und Grenznutzen gibt es nur für einzelne Faktoren und Güter. Und somit gibt es auch keine Sättigung der Bedürfnisse im ganzen. Wachstumsverzicht kann deshalb nicht die Botschaft einer Ökonomie sein, die (auch ökologische) Knappheiten und deren Überwindung zum Gegenstand hat.

Die Umsetzung der von den ökologischen Systemen unabweisbar gezogenen Begrenzungen einzelner Aktivitäten in wirtschaftlich wirksame ökologische Schranken hat nichts zu tun mit Grenzen des Wachstums oder gar mit einer Begrenzung von wirtschaftlicher Freiheit und Wohlstand. Die Limitierung der Inanspruchnahme von natïrlichen Produktionsfaktoren bedeutet keineswegs, dem System Wirtschaft den Atem zu nehmen. Denn jede Mengenbeschränkung wird in der Wirtschaft übersetzt in Änderungen relativer Preise und generiert somit einen Substitutionsprozeß. Schließlich ist es das ganz normale Geschäft der Wirtschaft, auf Änderungen relativer Preise mit im
Wettbewerb herauszufindenden intelligenten Anpassungen zu antworten. Da spielt es keine Rolle, ob diese Änderungen die Folge demokratisch legitimierter politischer Setzungen, technologischer Entwicklungen oder individueller Präferenzänderungen sind. Solange der homo oeconomicus gegebene Ziele mit geringerem Aufwand oder mit gegebenen Ressourcen weitergesteckte Ziele anstrebt und erreicht, muß es für das sozio-ökonomische System keine ,realen“ Wachstumsgrenzen geben.

Wem das gerade unter ökologischen Gesichtspunkten eher perspektivlos scheint, der sollte sich klar machen, daß der Weg in Richtung Nachhaltigkeit nichts anderes ist als Wachstum hinein in andere ökonomische Strukturen getrieben von anderen relativen Preisen. Einer solchen wirtschaftlichen Entwicklung sind keine Grenzen gesetzt.

\section{Geldpolitische Deckelung des ökologischen Strukturwandels}

Wohl aber gibt es unabhängig von Zielen und Inhalten des Wirtschaftens monetäre Grenzen des Wachstums. Eine arbeitsteilige Geldwirtschaft kann nur wachsen, sei es in Richtung Zerstörung der natïrlichen Lebensgrundlagen oder in Richtung Nachhaltigkeit, wenn die Geldpolitik die dafiur unerläßlichen Bedingungen schaftt. Hinsichtlich dieser Bedingungen nimmt die herrschende Lehre eine dogmengeschichtlich eher bescheidene Position ein. Die Rolle des Geldes wird reduziert auf die von Rechenpfennigen. Geld ist in bezug auf die reale Entwicklung neutral. Geldpolitik hat daher nur die Aufgabe, die zum Güterangebot passende Geldmenge zur Verfïgung zu stellen. Eine höhere Geldmenge fuihrt in der Logik dieser Theorie nur zur Finanzierung inflationärer Prozesse.
Konträr dazu ist beispielsweise bei Schumpeter Geld die treibende Kraft der Entwicklung, d.h. des Prozesses der ,schöpferischen Zerstörung“. Danach kann die Wirtschaft nur durch exogen bereitgestelltes zusätzliches Geld wachsen. Nur durch niedrige Realzinsen kann sie Wachstumsraten realisieren, die über den Produktivitätsfortschritt hinausgehen und die Arbeitslosigkeit abbauen (1). Angebotspolitik im Schumpeterschen Sinne heißt Angebot von fresh money, heißt Vorfinanzierung eines von Investitionen getragenen Aufschwungs durch die Zentralbank und damit auch Vorfinanzierung eines strukturellen Wandels in Richtung Nachhaltigkeit.

Eine potentialorientierte Geldpolitik, wie sie die Bundesbank seit 1974 betreibt und nachdrücklich der Europäischen Zentralbank empfiehlt, verhindert dies (2). Sie deckelt die Investitionsdynamik. Der Maßstab für die Geldversorgung ist die Entwicklung des gesamtwirtschaftlichen Produktionspotentials berechnet auf der Basis des Wachstumstrends der Vergangenheit, der in die Massenarbeitslosigkeit geführt hat. Für die beiden letzten Jahre ihrer Herrschaft über die nationale Geldversorgung (1997 und 1998) nimmt die Bundesbank dabei nur noch ein Potentialwachstum von durchschnittlich zwei Prozent an (3).

Doch was hier bescheiden als Annahme deklariert wird, ist in Wahrheit eine Vorgabe für das gesamtwirtschaftliche Wachstum. Wenn daher das Potentialwachstum wegen einer schwachen Investitionstätigkeit immer weiter herabgesetzt wird, dann muß sich das auch in den tatsächlichen Wachstumsraten niederschlagen. Eine höhere Wachstumsrate ist wegen der Finanzierungsschranke ausgeschlossen. Möglich sind aber konjunkturelle Einbrïche mit der Folge weiterer Absenkungen des Potentialtrends der Geldversorgung. So kommt es, daß der Sockel an Arbeitslosigkeit zu Beginn jeder neuen Aufschwungsphase höher ist als bei der vorhergehenden.

Falls sich diese Geldmengenorientierung für das „Euroland“ insgesamt durchsetzt (4) und ein Potentialwachstum in der Größenordnung von zwei bis zweieinhalb Prozent von der Europäischen Zentralbank unterstellt wird, dann gibt es bis auf weiteres in Europa nur ein wirtschaftliches Wachstum, das bestenfalls im Rahmen der Produktivitätsentwicklung liegt. Ein solches Wachstum aber reicht nicht aus, um die Massenarbeitslosigkeit zu überwinden. Daran kann keine rigorose Angebotspolitik, keine perma- 
nente Lohnsenkung mit deflationären Risiken, keine (auch ökologische) Steuerreform und kein staatliches Ausgabenprogramm etwas ändern.

Wenn daher der Begriff „Grenzen des Wachstums" einen Sinn macht, dann in bezug auf die von der potentialorientierten Geldmengenpolitik gezogene gesamtwirtschaftliche Finanzierungsschranke. Die Wirtschaft kann darauf nur mit Anpassungen bei den Investitionen reagieren, die auf noch niedrigere Wachstumsraten sowie noch bescheidenere Potentialtrends hinauslaufen und damit die Massenarbeitslosigkeit weiter verschärfen. Im Vergleich zu Umweltlizenzen/-zertifikaten bedeuten die monetären Lizenzen eine umfassende Deckelung der gesamtwirtschaftlichen Aktivitäten. Substitutionsprozesse, wie sie von Umweltzertifikaten und Umweltsteuern intendiert sind, kann es bei den Wachstumslizenzen der Zentralbank nicht geben.

So sehr die interessengeleitete Polemik gegen umweltpolitische Mengenschranken in Form von Lizenzen und Zertifikaten zurückgewiesen werden muß und Anspielungen auf Bezugsscheine und ökologische Zentralverwaltungswirtschaft in diesem Zusammenhang von Ignoranz beherrscht sein mögen, hier, bei der Geldpolitik, treffen sie allesamt auf das richtige Politikfeld. Die Volkswirte der „Zentralen Planungsbehörde" definieren das reale gesamtwirtschaftliche Plansoll, das Produktionspotential oder, in der DDR-Terminologie, die Aufkommensbilanz. Und die Zentralbank stellt die dafuir notwendige Geldversorgung bereit. Nach diesem Muster wird in Deutschland unter dem Beifall von Räten und Beiräten seit 25 Jahren Geldpolitik betrieben. Diese Politik war so erfolgreich, das inzwischen bei sinkenden Lohnstückkosten selbst die Zielin-

\section{Wollen Sie einen Überblick über die bisherigen Inhalie des Informations- dienstes "Ökologisch Wirtschaften"?}

Dann wählen Sie sich einfach ein unter http://www.umwelt.de/magazin/ oekowirt

Unter dieser Adresse können Sie zugleich auch bestellen! flationsrate der Bundesbank unterschritten wird und ein Abgleiten in die Deflation nicht mehr völlig auszuschließen ist. Dabei ist die Idee, die zukünftige Dynamik des entwicklungsoffenen komplexen Systems Marktwirtschaft in Potentialrechnungen abschätzen zu können, nichts anderes als „Anmaßung von Wissen" (Hayek). Hinzu kommt der tautologische Charakter solcher Rechnungen. Sie können empirisch nicht falsifiziert werden, weil sie über die Geldpolitik ihre Ergebnisse selbst herbeiführen.

\section{- Falscher ordnungspolitischer Imperativ}

Die Hinnahme der ordnungspolitischen Schieflage und der erkenntnislogischen Problematik der herrschenden Geldpolitik durch die Marktwirtschaftler steht in einem erstaunlichen Verhältnis zur ordnungspolitischen Sensibilität, mit der umweltpolitischen Vorstellungen begegnet wird. Das gilt besonders im Hinblick auf die Entwicklung von Leitbildern für Nachhaltigkeit. Hier ist die ordnungspolitische Dimension allgegenwärtig und es gilt der Anfangsverdacht eines latenten Abrutschens in Formen ökologischer Planwirtschaft. Doch ist die Gefahr von dieser Seite nicht das, was Ordnungspolitiker besonders umtreiben müßte.

Tatsächlich hat zwar die offizielle Umweltpolitik längst in einen fatalen ordnungspolitischen Dualismus hineingeführt. Danach gelten für private Güter die marktwirtschaftlichen Allokationsprinzipien und für das öffentliche Gut Umwelt das Prinzip des command and control. Für die Erhaltung der natürlichen Lebensgrundlagen „leisten“ wir uns ineffiziente Spielarten einer ökologischen Zentralverwaltungswirtschaft, während für private Güter effiziente Märkte eingesetzt werden.

Es scheint heute jedoch Konsens darüber zu herrschen, daß die Leitbilder für Nachhaltigkeit als ,regulative Idee“ für den die Zukunft gestaltenden gesellschaftlichen Suchprozeß und keinesfalls als politische Handlungsanweisungen aufzufassen sind.

Wäre es anders, was für eine Lawine von Schmähungen würde losgetreten werden, wenn etwa das ,Nachhaltigkeitspotential" Jahr für Jahr von einigen ökologen berechnet und via Umweltlizenzen gesteuert werden würde? Doch wenn die Zentralbank über die Emission von Geld, also über Lizenzen, die Investitionen kappt und den Beschäftigungszuwachs auf Null stellt, dann geschieht dies unter dem Beifall fast der gesamten Ökonomenszene.

Die monetäre Bremse an der europäischen Jobmaschine ist zugleich die Bremse für umweltpolitische Fortschritte. Ohne eine Perspektive für mehr Beschäftigung bleiben die Handlungsspielräume der Umweltpolitik extrem bescheiden. Und das gilt vermutlich für jede Regierung. Alle Regierungen stehen bei den heute noch vorherrschenden wirtschaftstheoretischen Mustern und der Massenarbeitslosigkeit unter dem Druck, die Privatökonomie auf vielfältige Weise zu entlasten. Die Umweltpolitik hat in diesem Spiel schlechte bzw. keine Karten. Umweltschutz ist nicht kostenlos zu haben, und zwar auch dann nicht, wenn das Aufkommen etwa aus Ökosteuern zur Entlastung an anderer Stelle eingesetzt wird. Deshalb müssen Umweltökonomen und Umweltpolitiker ein herausragendes Interesse am Lösen der monetären Bremse haben. Sie müssen sich auseinandersetzen mit der geldpolitischen Strategie der Europäischen Zentralbank, denn die monetären Grenzen des Wachstums bestimmen zugleich die politischen Grenzen ökologischer Möglichkeiten.

\section{Anmerkungen}

(1) Vgl. Carl-Ludwig Holffrerich, Gerhard Maier-Rigaud: Die monetäre Bremse an der europäischen Jobmaschine, Handelsblatt vom 4.6.1998.

(2) Vgl. zur potentialorientierten Geldpolitik meine beiden Aufsätze: Die Fiktion vom Produktionspotential, Wirtschaftsdienst, 1982/VII, S. 357-360 und

Der Zins, das Potential und der Aufschwung. Zur geldpolitischen Konzeption des Sachverständigenrates, Wirtschaftsdienst, 1983/I S. 45-50.

(3) Deutsche Bundesbank, Monatsbericht Januar 1998, Überprüfung der Geldmengenorientierung 1997/1998 und Konkretisierung des Geldmengenzieles für 1998, S. 19.

(4) Deutsche Bundesbank, Monatsbericht Januar 1998, Geldpolitische Strategien in den Ländern der Europäischen Union, S. 33-47.

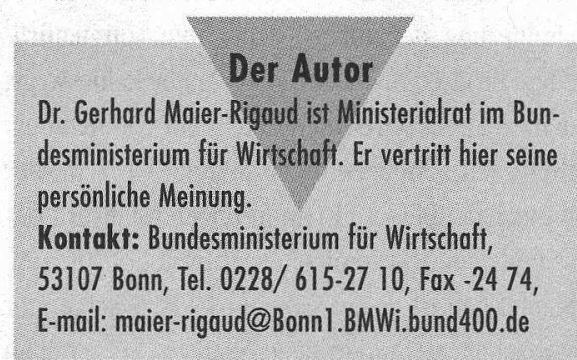


(c) 20I0 Authors; licensee IÖW and oekom verlag. This is an article distributed under the terms of the Creative Commons Attribution Non-Commercial No Derivates License (http://creativecommons.org/licenses/by-nc-nd/3.o/), which permits unrestricted use, distribution, and reproduction in any medium, provided the original work is properly cited. 